\title{
Les transgressions poétiques d'un auteur swahiliphone de Lubumbashi : Patrick Mudekereza
}

\author{
Flavia Aiello et Roberto Gaudioso
}

\section{(2) OpenEdition}

\section{Journals}

Édition électronique

URL : https://journals.openedition.org/coma/6157

DOI : $10.4000 /$ coma. 6157

ISSN : 2275-1742

\section{Éditeur}

Institut des textes \& manuscrits modernes (ITEM)

\section{Référence électronique}

Flavia Aiello et Roberto Gaudioso, «Les transgressions poétiques d'un auteur swahiliphone de Lubumbashi : Patrick Mudekereza », Continents manuscrits [En ligne], 15 | 2020, mis en ligne le 15 octobre 2020, consulté le 12 janvier 2023. URL : http://journals.openedition.org/coma/6157; DOI : https://doi.org/10.4000/coma.6157

Ce document a été généré automatiquement le 12 janvier 2023.

\section{cc) $(\mathrm{SP} \Theta$}

Creative Commons - Attribution - Pas d'Utilisation Commerciale - Pas de Modification 4.0 International - CC BY-NC-ND 4.0

https://creativecommons.org/licenses/by-nc-nd/4.0/ 


\title{
Les transgressions poétiques d'un auteur swahiliphone de Lubumbashi : Patrick Mudekereza
}

\author{
Flavia Aiello et Roberto Gaudioso
}

\section{Introduction}

En République Démocratique du Congo (RDC), le swahili, une des quatre langues nationales du pays ${ }^{1}$, joue le rôle de médium pour divers arts verbaux en prose et poésie. Les textes poétiques contemporains comprennent soit des chansons modernes soit des vers écrits, bien que ces derniers constituent aujourd'hui une production exiguë et souvent inédite, dans un contexte où l'écriture créative préfère la langue française. D'un point de vue historique, l'écriture créative congolaise s'est exprimée très tôt en langues autochtones, à la suite du prosélytisme missionnaire chrétien et de la réforme scolaire coloniale de 1925. Furent alors introduites dans le système éducatif les quatre langues véhiculaires (ciluba, kikongo, lingala et kiswahili), ainsi que d'autres parlers de grande diffusion (lomongo, otetela, kinande, etc.), ce qui conduisit les prêtres, catéchistes et élèves congolais ${ }^{2}$ à composer des contes, hymnes et poèmes dans leur propres langues africaines ${ }^{3}$. Toutefois, depuis le lendemain de la Seconde Guerre mondiale, la production congolaise écrite en français s'est développée à tel point que la définition même de « littérature congolaise » dans la perception commune renvoie de facto à la "littérature congolaise d'expression française ${ }^{4}$. Les publications dans les langues véhiculaires devenues après l'Indépendance les quatre langues nationales ${ }^{5}$ sont largement inférieures en nombre (moins de $10 \%$ ) par rapport aux œuvres écrites en français aussi bien en RDC que dans la diaspora ${ }^{6}$.

Des productions en langues africaines congolaises continuent néanmoins à paraître ${ }^{7}$, quoique peu visibles, notamment en langue swahili. Le genre théâtral, dans lequel, à l'époque coloniale, le swahili a été parfois utilisé à l'écrit ${ }^{9}$, s'est par la suite développé au niveau de la performance, essentiellement orale ${ }^{10}$; un seul conte ${ }^{11}$, davantage de 
poésie, même si la publication a souvent eu lieu à l'étranger, et les ouvrages n'ont vraisemblablement pas beaucoup circulé en RDC. Dans les dernières décennies ont paru divers recueils poétiques publiés par les écrivains swahiliphones, parmi lesquels deux auteurs du Kivu, Jean-Robert Kasele Laisi Watutwa ${ }^{12}$ et Charles Djungu-Simba ${ }^{13}$, ou encore le poète Huit Mulongo Kalonda Ba-Mpeta de Lubumbashi ; tous sont professeurs des universités. Ces créations, qui témoignent de l'importance du monde artistique swahiliphone, liée à la diffusion progressive du swahili de la côte (pwani) jusqu'aux régions continentales (bara) de l'Afrique orientale et centrale au cours des deux derniers siècles ${ }^{14}$, ont rarement fait l'objet d'étude des critiques de la littérature swahilie moderne et contemporaine, plutôt focalisées sur les textes de nombreux auteurs du Kenya et de la Tanzanie. Par ailleurs, d'autres poèmes en swahili restent pour le moment inédits, comme ceux de Patrick Mudekereza, directeur du centre culturel WAZA à Lubumbashi. Dans cette contribution, après une introduction sur la complexité linguistique du contexte swahiliphone de Lubumbashi, et sur la poésie d'expression swahilie au Katanga, nous présenterons une analyse textuelle et comparative des compositions de Mudekereza, qui se centrera sur la transgression linguistique et physique présente dans ses vers.

\section{Le contexte swahiliphone de Lubumbashi}

La situation linguistique du swahili au Katanga est particulièrement complexe. Le développement de cette langue exogène et les mouvements migratoires caractérisent depuis toujours les milieux urbains de la région, au point qu'on pourrait parler de viswahili (pluriel de kiswahili, "langue swahili») à Lubumbashi, c'est-à-dire de différents types de swahili parlés dans le même contexte ${ }^{15}$. Parmi ces variantes en contact permanent on pourrait mentionner la variété locale de Lubumbashi, le swahili des autres zones swahiliphones dans l'est du Congo (Maniema, Kivu etc.), beaucoup plus proche du swahili parlé en Tanzanie, et des formes "améliorées» de swahili sur le modèle du swahili standardisé de l'Afrique orientale ${ }^{16}$. Celles-ci, nées durant la période coloniale dans le milieu religieux et scolaire, sont souvent qualifiées de kiswahili bora (« excellent, meilleur swahili »).

La variété véhiculaire du swahili qui émergea dans le contexte de l'exploitation minière du Katanga à l'époque coloniale ${ }^{17}$ est devenue, au cours des générations, la langue principale de la population de Lubumbashi, et parfois sa langue maternelle ${ }^{18}$. En considérant son parcours historique, on comprend pourquoi, bien que partageant certains traits généraux avec les autres variantes de swahili du Congo, le swahili de Lubumbashi présente une physionomie plutôt autonome et, comme toute langue émergente urbaine, un important métissage linguistique ${ }^{19}$, par exemple dans le lexique, riche en emprunts des autres langues bantu de la région, et aussi du français et de l'anglais. Ce métissage est lié à son développement essentiellement oral, le swahili étant utilisé principalement dans la communication quotidienne et dans certaines formes de créativité artistique à diffusion verbale (comme la chanson et le théatre populaire) ${ }^{20}$. À cause du multilinguisme des locuteurs, ces variantes sont souvent caractérisées par les phénomènes de code-switching et code-mixing avec d'autres langues locales, le français et le lingala.

Des écrits en swahili ont paru au Katanga depuis l'époque coloniale (journaux, lettres, textes religieux, autobiographies, œuvres dramaturgiques), influencés à des degrés 
divers par le kiswahili bora. Pendant cette période, notamment après la réforme scolaire de 1925, les quatre langues véhiculaires (ciluba, kikongo, lingala et swahili) avaient une large place dans l'éducation de base. Mais peu après l'indépendance du pays, l'ordonnance $n^{\circ} 174$ du 17 octobre 1962 qui donna lieu au Programme de 1963 imposa le français comme langue d'enseignement au cycle primaire ${ }^{21}$. En dépit des directives linguistiques, établies à ce moment, pour faciliter l'adoption des langues véhiculaires régionales comme langues de l'enseignement, dans un cadre du système éducatif très critique, avec un taux élevé d'analphabétisme (masculin 17,5\%, féminin $45,9 \%$ en aires urbaines) et $15 \%$ d'accès aux secondaires, les ressources n'ont jamais été allouées afin que le swahili devienne la langue d'instruction dans les territoires swahiliphones. Il reste ainsi un enseignement marginal, limité aux premières années de l'école primaire, et avec des enseignants peu formés ${ }^{22}$. Patrick Mudekereza a lui-même témoigné n'avoir eu quasiment aucune formation scolaire en swahili ${ }^{23}$. La question de l'enseignement du swahili connait régulièrement un regain d'actualité, surtout ces dernières années, lorsque les grands organismes internationaux comme l'USAID (United States Agency for International Development) ou le DFID (Department for International Development, UK) ont estimé fondamental d'insérer dans les programmes de développement de la RDC l'enseignement en langue "locale» dans les écoles primaires. À partir de 2017, le DFID, en collaboration avec l'USAID, a lancé le programme ACCELERE ! avec l'objectif de former les enseignants et d'insérer des heures de langue swahilie dans les classes primaires du Katanga, dans le but d'améliorer l'accès, la qualité et la gouvernance dans l'enseignement primaire de la $\mathrm{RDC}^{24}$. On doit toutefois préciser que le kiswahili bora ne constitue pas du tout une norme linguistique bien définie au Katanga et, plus largement en RDC. Il reste une catégorie nébuleuse diversement associée au swahili standard de l'Afrique orientale, à celui parlé en Tanzanie, à celui de l'est du Congo, au swahili utilisé dans les églises et dans les écrits religieux, ainsi qu'à celui employé par les médias (radio et télévision) ${ }^{25}$. Son usage correspond, de fait, à divers degrés de négociation entre le swahili standard et les principales caractéristiques communes à ses variantes congolaises.

Notons que les locuteurs de Lubumbashi distinguent clairement le registre familier et le registre formel, essentiellement écrit, avec sa norme et son corpus littéraire (français, congolais et d'Afrique francophone) opposé aux arts «populaires» qui, eux, s'expriment en swahili local. Le recours au swahili sous sa forme écrite présente un modèle linguistique plutôt incertain et flou, en raison même de la situation décrite plus haut. Il y a moins de productions textuelles de référence accessibles (mis à part les écrits de nature didactique ou informative publiés par les éditions religieuses ${ }^{26}$ ), surtout en ce qui concerne l'écriture créative. Les rares publications littéraires congolaises en swahili restent peu accessibles dans les librairies ou bibliothèques, et c'est plus vrai encore des œuvres en swahili de l'Afrique orientale, conséquence de la difficile compréhension, de la distance linguistique et de l'absence d'usage scolaire de la littérature swahilie classique ou moderne.

C'est dans ce contexte linguistique particulier que se situe la production poétique en langue swahilie au Katanga, présentée dans les pages qui suivent. 


\section{Poésie swahilie au Katanga}

Au Katanga, et en particulier à Lubumbashi, où les auteurs de l'article ont mené une recherche de terrain dans le cadre d'un projet d'intérêt national (PRIN) intitulé "Mobilité - stabilisation. Représentations congolaises et dynamiques sociales, au Congo et dans l'espace global $^{27} "$, existent aujourd'hui différentes productions poétiques en swahili, qui comprennent chansons et poèmes écrits. Parmi les chansons signalons les textes de l'auteur-compositeur Sando Marteau ${ }^{28}$, dans lesquels émergent les réflexions existentielles ${ }^{29}$, la valorisation artistique du kiswairi (kiswahili dans la prononciation locale ${ }^{30}$ ), parfois mélangé à des éléments de kiswahili bora ${ }^{31}$, et le sens engagé du travail de l'artiste ${ }^{32}$, exprimé par exemple dans la chanson «Magumu na shida $^{33} »$. Le chanteur y rapporte avec déception et résilience l'agression subie pendant un concert dans une autre ville, évoquée sous un nom fictif :

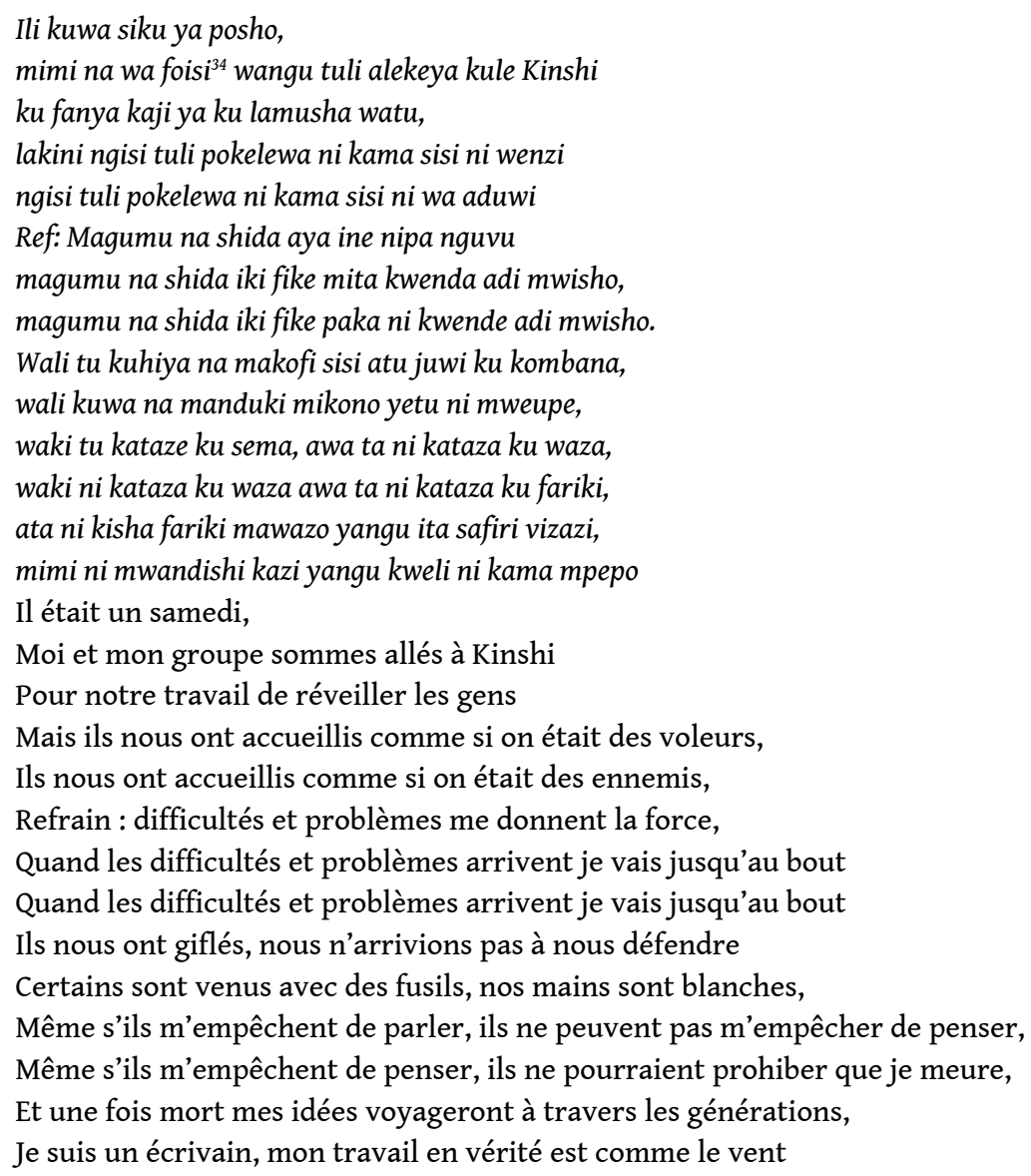

En ce qui concerne les vers écrits, l'auteur le plus connu est Huit Mulongo Kalonda BaMpeta, né en 1955 à Lubumbashi; il est poète, romancier, dramaturge, critique littéraire et activiste politique. Professeur à l'Université de Lubumbashi (UNILU) depuis 1990, année de son retour après une longue période en France (où il a obtenu un doctorat en langue et littérature française), il est l'auteur de deux recueils poétiques en swahili, Uhuru (Liberté/Independence, 1990) et Utenzi (Poème, 1990), publiés à Paris ${ }^{35}$, et de poèmes en swahili, lingala et kiluba, présents dans la plaquette francophone Pleurs éternelles (2006) sortie à Lubumbashi ${ }^{36}$. Toutefois, ses œuvres en swahili, du fait qu'elles ont été publiées à l'étranger, ne sont pas aujourd'hui disponibles en $\mathrm{RDC}^{37}$. Nkashama ${ }^{38}$ cite un seul texte pris de Utenzi, dans lequel on note l'usage du swahili 
bora, forme proche du swahili standard de l'Afrique orientale, lequel, comme signalé plus haut, est associé aux registres les plus élevés, de nature religieuse ou éducative :

\begin{tabular}{|l|l|}
\hline Furaha wangu adui & La joie mon ennemi \\
\hline Mawazo wangu ndugu & Les pensées mes frères \\
\hline Natazama tena hile siku & Je regarde encore ce jour-là \\
\hline Natembea sawa askari & Je marche tel un soldat \\
\hline Natembea sawa mw'elimu & Je marche tel un maître \\
\hline Natembea sawa mzimu & Je marche tel un esprit \\
\hline
\end{tabular}

Les textes swahilis de Huit Mulongo Kalonda Ba-Mpeta constituent un cas isolé par rapport au reste des publications de poésie au Katanga, toutes francophones, qui ont considérablement augmenté dans les dernières trois décennies, à partir de la fin du régime de Mobutu, avec des auteurs tels que Kilanga Musinde, Cidibi Ciakandu, Wenu Bekere, Tshisungu wa Tshisungu, Mutonkole Lunda, Mutenke Ngoy Maïte, Selemani Ngongo, Maguy Kabongo et Jano Bakasanda ${ }^{39}$. Il s'agit d'une production poétique qui, même dans ce cas, naît dans le milieu académique et, sur les traces de la génération de Valentin-Yves Mudimbe, Julien Kilanga et d'autres poètes formés à l'Université de Lubumbashi. Elle utilise, surtout à son début, un français très recherché, avec des références à la culture classique et des formes d'expérimentation chères aux fondateurs de la Négritude ${ }^{40}$. Très présents aussi, les thèmes socio-politiques, comme dans les recueils en langue swahilie, et l'expression d'une identité katangaise, antithétique au pouvoir central, spécialement durant la dictature de Mobutu, dont on dénonce les abus et les violences (comme le massacre des étudiants à l'Université de Lubumbashi en 1990), et dont la destitution par Laurent D. Kabila est saluée comme une libération ${ }^{41}$. Plusieurs initiatives ont été entreprises durant les dernières années pour élargir la diffusion et la réception de la poésie katangaise, avec l'aide de l'université et des écoles secondaires, des bibliothèques et du cercle "Cellule littéraire de Lubumbashi", qui organise annuellement Les journées littéraires de Lubumbashi ${ }^{42}$. Toutes ces activités sont destinées à la promotion de la poésie francophone katangaise, et nombre de productions poétiques en swahili restent pour le moment inédites. Durant notre présence à Lubumbashi, nous avons rencontré Sumba Maly, qui nous a gentiment donné à lire son manuscrit Uhai (Vie), ainsi que Patrick Mudekereza, mais il n'est pas exclu que d'autres auteurs « invisibles » se trouvent dans ce contexte swahiliphone.

Les vers de Sumba Maly semblent s'inscrire dans la lignée de Huit Mulongo Kalonda BaMpeta, qui se rattache à la communauté swahiliphone de l'Afrique orientale par son usage du kiswahili bora et des références littéraires, par exemple le titre du recueil Utenzi, terme qui indique un genre prestigieux de la tradition poétique swahilie, à savoir de longs poèmes narratifs diffusés le long de la côte swahilie, soit sous forme orale, soit à travers les manuscrits en caractères arabes (ajami). Dans les poèmes de Maly, on remarque, outre l'utilisation d'une forme de swahili plus proche du standard, plusieurs toponymes de Tanzanie, éléments visant «à revendiquer l'appartenance à la communauté swahilie et aux sources tanzaniennes qu'il exalte dans plusieurs de ses 
poèmes ${ }^{43}$ ». Un bon exemple est le poème Sina kikao, avec les toponymes "Tanga », « Namanga », "Tanganika », où on remarque aussi la propension de l'auteur aux jeux verbaux :

\begin{tabular}{|c|c|}
\hline Na tanka-tanka & J'erre \\
\hline Sina kikao & Je n'ai où demeurer \\
\hline Toka Busanga & Depuis Busanga $^{44}$ \\
\hline Kwenda Tanga & Vers Tanga \\
\hline Toka Katanga & Depuis le Katanga \\
\hline Kwenda Namanga & Vers Namanga \\
\hline Wapi kuna Kisanga & Où est Kisanga, l'île ${ }^{45}$ \\
\hline Cha maisha? & De la vie? \\
\hline Na danganika & Je m'illusionne \\
\hline Kama Tanganika & Comme le Tanganyika \\
\hline Kuna alama & Il y a un signal \\
\hline Sina salama & Je ne suis pas en sécurité \\
\hline Na lalamika! & Je crie! \\
\hline Na lalamika! & Je crie! \\
\hline Nikilala shamba & Si je dors aux champs ${ }^{46}$ \\
\hline Na itwa mshamba & On m'appelle paysan \\
\hline Niki lala mjini & Si je dors au village \\
\hline Ni kama chini & C'est comme (dormir) à même le sol \\
\hline Ni kitenda mazuri & Si je fais de bonnes actions \\
\hline Ninaitwa mabayi & On m'appelle méchant \\
\hline Na tanka-tanka! & J'erre! \\
\hline Na tanka-tanka! & J'erre! \\
\hline Na tamani kuishi & Je veux vivre \\
\hline Na imani kuishi & Avec la foi, vivre \\
\hline Kwa amani alisi & Dans une véritable paix \\
\hline Kati-kati ya mupendo & Au cœur de l'amour \\
\hline Inchi ya salama. & Dans un pays en sécurité \\
\hline
\end{tabular}

En comparaison avec les poètes swahiliphones de Lubumbashi mentionnés plus haut, Patrick Mudekereza se distingue pour son rapport différent à la langue swahilie. Il n'est pas influencé par la référence au modèle du kiswahili bora, ni par le rattachement à la communauté swahilie de l'Afrique orientale et à son monde littéraire, et sa création acquiert une dimension très personnelle et intime. 


\section{La transgression dans les vers de Patrick Mudekereza $^{47}$}

Né à Lubumbashi en 1983, Patrick Mudekereza est le directeur du centre d'art WAZA ${ }^{48}$ qui se trouve au centre-ville de Lubumbashi. Bien qu'il ait commencé dès son adolescence à écrire des poèmes et des textes courts, comme il nous l'a raconté lors d'une interview à Lubumbashi (septembre 2018), Patrick Mudekereza n'a jamais publié ses poèmes dans des recueils poétiques. Il considère son écriture essentiellement comme une pratique associée à son travail dédié à l'art. Ses textes ne sont pas de simples commentaires de l'œuvre des artistes avec lesquels il collabore, mais des réflexions originales en interaction avec l'art, et souvent en dialogue avec ses chercheurs ${ }^{49}$. Écrivain engagé dans plusieurs formes d'écriture, créative et discursive (cette dernière en français ou anglais), Patrick Mudekereza semble avoir un rapport très particulier et intime à la poésie, qu'il pratique de manière intermittente et dont il ne garde pas trace de façon systématique. Quelques-unes de ses créations ont été présentées en public, par exemple celles composées durant les travaux du collectif d'écrivains Libr'Écrire, né à Lubumbashi en 2007 (https://www.centredartwaza.org/? portfolio=librecrire ) et qui se réunit jusqu'en 2010.

Mudekereza nous a confié six textes, dont trois (Funga macho liya, Pima tuyambe et Langues) ont été composés dans le contexte du collectif Libr'Écrire, deux ne sont pas datés (Buzuri tele et $\mathrm{Mu}$ pori ya binvuri [Dans le bois des ombres]), et un (Mankamanshi>Mboleo [Morve>Fertilisant]) est daté du 13 février 2018. Trois de ces compositions poétiques sont bi/multilingue, en français, swahili et lingala (Funga macho liya, Langues, Mankamanshi>Mboleo), les autres sont écrites en swahili.

Ce sont des textes qui n'ont pas un genre bien défini, où Mudekereza donne libre cours à sa liberté créative. Ainsi dans Funga macho, liya, où les vers en swahili sont placés côte à côte avec le français, et suivis par des lignes de prose en français :

\begin{tabular}{|l|l|}
\hline Funga macho, liya & Ferme les yeux, pleure \\
\hline Machozi itasafisha macho & Les larmes purifient les yeux \\
\hline Funga macho, lota & Ferme les yeux, rêve \\
\hline Nvula itasafisha machozi & La pluie purifie les larmes \\
\hline
\end{tabular}

C'est dans ma ville que le ciel est le plus bleu. Quand il pleut, nous levons nos mains colorées par nos travaux pour former un arc-en-ciel. Mains blanches de meuniers, mains vert malachite de mineurs, mains rouges de cultivateurs, mains noires de cireurs, etc. Des milliers de petites mains levées vers le ciel accueillent la pluie qui ensemence notre force vitale. Et mes mains multicolores de choqueurs ${ }^{50}$ se lèvent aussi. Si elles peuvent arrêter la pluie, elles pourront aussi arrêter la fatalité de notre misère.

Dans le poème, Mudekereza réaffirme en français ce qu'il affirme en swahili. Et le texte en prose qui suit, écrit en français, travaille toujours un registre corporel, concret. La ville de Lubumbashi, avec son passé et son présent, s'identifie aux corps de ses travailleurs. Dans les travailleurs, il y a le beau, les couleurs, l'arc-en-ciel. En ce sens, l'écrivain nous rappelle Jean Genet, un auteur aimés1, qui voyait le haut dans le bas et le bas dans le haut ${ }^{52}$. L'image dans les dernières lignes sur la condition des « choqueurs » 
qui se débrouillent dans le quotidien est aussi forte que dure. Le poète crée ici une phrase hypothétique irréelle - bien entendu, personne ne peut arrêter la pluie, c'est cela qui donne un trait d'ironie et de désespoir. Si personne ne peut arrêter la misère, nous avons encore besoin (peut-être pour toujours) de l'écart cognitif de la poésie, qui nous fait reconnaître "notre misère", et pas seulement l'humble condition des travailleurs. La beauté des vers de Mudekereza est liée à une dimension très corporelle et souvent intime, comme dans le poème Buzuri tele (Beauté pleine), où le beau coïncide avec la corporalité, l'éros et l'extase dionysiaque :

\begin{tabular}{|l|l|}
\hline Buzuri yake machafu tele & Sa beauté emplissant les joues \\
\hline Cheko yake inaivuyisha & Son sourire fait transpirer \\
\hline Roho yangu naya inavuya vile & Mon âme qui transpire pareillement \\
\hline Na miye wote inalobanisha & Et me mouille tout entier \\
\hline Chekeni chekeni & Riez, riez \\
\hline Mwanya ya meno yake & L'interstice entre ses dents \\
\hline Nyesheni buzuri yote & Montrez toute la beauté \\
\hline Chezeni chezeni & Jouez, jouez \\
\hline Minofu ya roho yangu & La bonne chaire de mon âme \\
\hline Mapendo ni feti, & L'amour est une fête \\
\hline Leweni & Soûlez vous \\
\hline
\end{tabular}

Tous ces éléments, l'éros et la poésie, la transgression et la liberté sont rendus plus intenses dans le poème Pima tuyambe (« Ose, et allons chier ! ») :

\begin{tabular}{|l|l|}
\hline Pima tuyambe! & Ose, et allons chier ! \\
\hline Tuende ngambo ingine ya mulima & Allons de l'autre côté de la montagne \\
\hline Kule mecho na kinywa inaimba & Là-bas, les yeux et la bouche chantent \\
\hline Shangwe ya uhuru53 & La joie de la liberté \\
\hline Leta tuondje! & Amène, qu'on goûte! \\
\hline Anika ngozi fasi yote & Etends la peau partout \\
\hline Tukigusa, tugusane & Si nous touchons, touchons-nous l'un l'autre \\
\hline Tukiona, tuonane & Si nous voyons, voyons-nous l'un l'autre \\
\hline
\end{tabular}




\begin{tabular}{|l|l|}
\hline Tukikula, tushibane & Si nous mangeons, rassasions-nous l'un l'autre \\
\hline Panda tukomeshe! & Plante, (et) faisons croître! \\
\hline Tupandane! & Montons-nous ${ }^{54}$ l'un sur l'autre! \\
\hline Tuoteshe yetu shirika & Faisons pousser notre relation \\
\hline Ukitema jacho, niliye mikoyo & Si tu craches la sueur, que je pleure urine ${ }^{55}$ \\
\hline Ukikula yangu tunda, nilewe yako majiba & Si tu manges de mon fruit, que je m'enivre de ton \\
\hline Hara, tuyambe & Purge-toi, allons chier \\
\hline Tusafishe ndani mwetu & Nettoyons notre intérieur \\
\hline Yamba mawazo ya uchungu & Chie les pensées douloureuses \\
\hline Kumbusho ya vita & Le souvenir de la guerre \\
\hline Hara, tujaze raha & Purge-toi, remplissons de plaisir \\
\hline Muda ilobanishe milele. & Que le temps mouille pour toujours \\
\hline
\end{tabular}

Ce poème a un rythme très accéléré, créé par une certaine régularité interne des strophes centrales. En particulier, on remarque que dans la deuxième strophe les vers 3 à 5 comptent huit syllabes avec une césure au centre, une rime initiale en " tu » soit dans le premier soit dans le deuxième hémistiche, une assonance des voyelles finales du premier hémistiche en « $a$ » et une rime finale dans le deuxième hémistiche en " ane ». Les premiers vers de la strophe suivante prolongent l'assonance en «tu». Dans la strophe initiale et finale le rythme est plus détendu, le moi lyrique invoque un autre corps et invoque la dépuration. L'assonance entre hara («purge-toi») e raha (" plaisir») réaffirme, enfin, que l'élément fondamental est le plaisir. Il s'agit d'un corps en mouvement, comme emphatisé par le rythme, dansant et aimant. Le plaisir et le sexe sont le moyen et le but de la "fête de la liberté », parce que le moi lyrique invite à aller de l'autre côté de la montagne, où seront chantés la fête de la liberté et l'extase qui la suit. En même temps, l'extase érotique sert à se purger - «Purge-toi, allons chier [...] chie les pensées douloureuses/le souvenir de la guerre » - à faire en sorte qu'il y ait vraiment la fête de la liberté.

On retrouve le même processus dans les poèmes du poète tanzanien Euphrase Kezilahabi, surtout dans ses créations des années 1980 contenues dans le recueil Karibu $\mathrm{Ndani}^{57}$, où les éléments corporels sont principalement liés à l'extase de la danse qui comprend éros et thanatos, la mort douloureuse, le tonicum purgeant ${ }^{58}$. La différence entre les années 1980 et 1990 est qu'en 1990 l'écriture se fait orphique et en quelque sorte romantique, invoquant la magie du rite et de la poésie pour arriver à une palingenèse de l'humanité.

Dans l'œuvre de Mudekereza, l'élément orphique de la fête et de l'éros reste plus concret et individuel. La description d'un rapport sexuel, même exprimée avec une 
polysémie raffinée (le double sens des mots -panda, mikoyo, majiba) reste précise, tandis que, dans le vers de Kezilahabi, on trouve rarement ce type de descriptions ; elles coïncident souvent avec l'extase de la danse ou sont tout simplement métaphorisées. Par ailleurs, c'est seulement dans les poèmes de sa dernière plaquette Dhifa ${ }^{59}$ que Kezilahabi adopte un langage familier, comme dans le poème Mke wa Waziri (Wimbo wa Harusi) ${ }^{60}$.

Huyo mumeo anatoka kijiji jirani Tulimwona akilambwa na mbwa Alipomaliza kujisaidia
Ton mari vient du village voisin

Nous l'avons vu être léché par un chien

Après s'être soulagé

Dans ce recueil, Kezilahabi est désormais conscient de la place de sa poésie dans la société, par rapport à la communauté des poètes, par rapport aux religions ; ainsi dans le poème Kuwako ${ }^{61}$, par exemple, le moi lyrique met en garde Dieu :

\begin{tabular}{|l|l|}
\hline Nitashtakiwa kwa yale niliyoyatenda uhuruni & Je serai accusé pour tout ce que j'ai fait en \\
Na kwa ushairi wangu mbaya umfanyao akae & liberté \\
macho. & Et pour ma mauvaise poésie qui le fera rester \\
& éveillé. \\
\hline
\end{tabular}

Dans les deux cas, la transgression est nécessaire pour la libération, et la libération reste le but ultime. Ceci n'est pas une capitulation de la poésie, non plus une défaite de la poésie envers le beau, bien au contraire. Le pouvoir de la poésie, qui ne se rétracte jamais dans les conditions les plus difficiles, imprègne notre existence jusqu'à nos déchets, notre misère, notre souillure. Ces éléments sont quasiment une nouveauté totale dans la poésie swahilie, à l'exception du dernier recueil du poète tanzanien Euphrase Kezilahabi, où il emploie souvent un registre bas. Par exemple, dans le poème Mafuriko ${ }^{62}$ les éléments bas constituent la poésie :

\begin{tabular}{|l|l|}
\hline Nitaandika wimbo juu ya mbawa za nzi & Je vais écrire une chanson sur les ailes d'une mouche \\
Utoe muziki arukapo wausikie walio & Qui en volant puisse porter la musique à beaucoup de \\
wengi & gens \\
Ushairi wa jalalani utaimbwa & La poésie de la décharge sera chantée \\
Juu ya vidonda vya wakulima & Sur les plaies des paysans \\
Na usaha ulio jasho lao. & Et le pus qu'ils transpirent \\
\hline
\end{tabular}

Comme on vient de le souligner, il ne s'agit pas d'un retrait de la poésie, bien au contraire, comme si la poésie pouvait extirper le mal, plutôt qu'en être affectée.

Dans la description d'expériences limites, les deux poètes semblent avoir à l'esprit la leçon d'Arthur Rimbaud ${ }^{63}$ qui avec le poème « Le cœur du pitre » exprime une violence sexuelle; dans la lettre qui accompagnait le poème il disait vouloir devenir un poète voyant $t^{64}$.

Mon triste cœur bave à la poupe,

Mon cœur couvert de caporal :

Ils y lancent des jets de soupe,

Mon triste cœur bave à la poupe :

Sous les quolibets de la troupe 
Qui pousse un rire général,

Mon triste cœur bave à la poupe,

Mon cœur couvert de caporal!

Ithyphalliques et pioupiesques

Leurs quolibets l'ont dépravé !

Au gouvernail on voit des fresques

Ithyphalliques et pioupiesques.

ô flots abracadabrantesques,

Prenez mon cœur, qu'il soit lavé !

Ithyphalliques et pioupiesques

Leurs quolibets l'ont dépravé !

Quand ils auront tari leurs chiques,

Comment agir, ô cœur volé ?

Ce seront des hoquets bachiques

Quand ils auront tari leurs chiques :

J'aurai des sursauts stomachiques,

Moi, si mon cœur est ravalé :

Quand ils auront tari leurs chiques,

Comment agir, ô cœur volé ?

Si la poésie ne se rétracte pas, mais se fait encore plus voyante, la transgression reside donc dans la volonté de voir l'expression poétique accéder à la force créatrice et purificatrice de la poésie.

La transgression dans l'écriture de Mudekereza n'est pas un aspect secondaire, mais un élément auquel le poète de Lubumbashi aspire consciemment. Mudekereza, en fait, nous a dit ${ }^{65}$ qu'il a été influencé par deux productions, toutes les deux porteuses de transgression : l'auteur-chanteur George Brassens et les chants funèbres zangazanga du Katanga. Brassens, sans surprise, est l'auteur transgressif qui chantait d'être un «pornographe » :

Tous les samedis, je vais à confesse

m'accuser d'avoir parlé de fesses

et je promets ferme au marabout

de les mettre tabou.

Mais

craignant, si je n'en parle plus

de finir à l'Armée du Salut,

je remets bientôt sur le tapis

les fesses impies.

Je suis le pornographe

du phonographe,

le polisson de la chanson.

Ma femme est, soit dit en passant

d'un naturel concupiscent

qui l'incite à se coucher nue

sous le premier venu.

Mais

m'est-il permis, soyons sincères,

d'en parler au café-concert

sans dire qu'elle a, suraigu

le feu au cul?

Le fait que l'auteur français soit sa principale référence poétique nous a amené encore davantage à considérer le texte Funga Macho, liya comme une double traduction sans original, parce que dans l'œuvre de Mudekereza français et swahili sont les langues de l'existence quotidienne, auxquelles il a recours pour la création poétique. Son swahili 
n'exprime aucune tentative de se conformer au modèle du swahili bora, il reste plutôt l'expression de son vécu. Il lui arrive bien sûr d'écrire uniquement dans l'une des deux langues, comme dans la vie il lui arrive aussi de s'exprimer seulement dans l'une ou l'autre langue selon les contextes, ou encore de les mélanger. Il est clair que la conscience politique (postcoloniale) de Mudekereza ne s'interprète pas comme une simple équation, un refus de la langue française comme symbole du colonialisme. Mudekereza est loin des poses idéologiques, il ne fait pas du français un symbole, mais un élément productif de sa réalité. Cela n'enlève rien à sa conscience politique, à son engagement pour promouvoir sur son territoire la création artistique congolaise et africaine, en encourageant notamment la création lushoise, et en valorisant la variante locale de swahili.

L'autre point de référence du poète de Lubumbashi sont les chants d'enterrement zangazanga, durant lesquels prime une liberté absolue ; on peut tout dire pendant que le corps est amené au cimetière. Joël Noret et Pierre Petit définissent comme "humoristique et obscène " le caractère des chants zangazanga ${ }^{66}$, surtout avant l'enterrement.

Après l'enterrement, les zangazanga peuvent encore rester présents sur les lieux du deuil, mais de façon un peu plus discrète. En échange de leur prestation, ils réclament de l'alcool lutuku, fument parfois du chanvre et entonnent des chants profanes. Ils font en quelque sorte contrepoids à la présence chrétienne, et n'hésitent pas à imiter les pasteurs avec beaucoup d'ironie.

Noret et Petit ont établi qu'à l'origine le terme zangazanga se référait seulement aux chants, puis que des groupes de jeunes introduisirent dans les années 1990 cette pratique importée de Kinshasa à Lubumbashi, initialement dans le quartier Kenya. Noret et Petit affirment que les chants de Lubumbashi sont moins violents que ceux de Kinshasa, mais on ne sait pas sous quels aspects ils ont été influencés par la spécificité $\mathrm{du}$ lieu. Nous pouvons supposer qu'au moins au niveau des textes, en adaptant aussi la langue, il y a eu une influence des chants populaires préexistants. Il serait fort intéressant d'approfondir les particularités des zangazanga et l'interaction avec l'autre musique de Lubumbashi, étant donné que dans le contexte des funérailles à Lubumbashi on note deux traditions musicales: zangazanga et kalindula ${ }^{67}$. Patrick Mudekereza a fait aussi référence dans nos conversations au genre kalindula, affirmant beaucoup apprécier cette forme musicale. Les groupes kalindula ${ }^{68}$ ont commencé à être populaires à Lubumbashi au cours des années $1970^{69}$, donc une vingtaine d'années avant les zangazanga. Noret et Petit remarquent une différence entre les deux groupes: les zangazanga produisent principalement des chants obscènes, tandis que les kalindula ont un répertoire plus vaste en genres et en registres ${ }^{70}$, comme l'affirment aussi Donatien Dibwe dia Mwembu et Claude Mwilambwe Mwende ${ }^{71}$. Dans les interviews conduites par ces deux derniers spécialistes, les enquêtés ont donné leurs avis sur les performances de kalindula. Ils ne sont pas très différents de ceux émis sur les chansons zangazanga, à savoir : une musique provocatrice et obscène, au point que Dibwe dia Mwembu et Mwilambwe Mwende concluent en mettant l'accent sur ce concept et donnent une valeur de dénonciation sociale aux performances des kalindula, puisque, de façon satirique et provocatrice, elles font émerger de manière directe la "vérité toute crue » :

Elle se caractérise aussi par une structure non raffinée de chansons spontanées, adaptées à la situation ambiante. La musique kalindula n'a de l'importance, selon l'opinion publique, que par rapport à certains événements heureux ou malheureux. Les orchestres kalindula regorgent d'artistes musiciens autodidactes qui, à l'instar 
des peintres populaires, crachent la vérité toute crue. Ils sont satiriques et font rire. Ils moralisent, prodiguent des conseils à qui veut les entendre. Ils savent consoler, détendre l'atmosphère lors des cérémonies de deuil, ils s'en prennent à l'attitude des parents ${ }^{72}$.

Kalindula et zangazanga font partie de la double tradition textuelle transgressive à laquelle a été exposé Patrick Mudekereza : Brassens et zangazanga! Le poète devient pornographe et chanteur de la vulgarité du quotidien, parce que, comme chez Kezilahabi, la poésie ne s'éloigne pas des moments difficiles de notre existence; au contraire, elle en sort renforcée en montrant une forme de liberté qui nous touche du point de vue émotionnel et cognitif. Pour Kezilahabi, la littérature africaine devrait être libératrice, c'est-à-dire se fixer pour but de libérer les Africains de la colonisation mentale provenant de l'extérieur, du moralisme, du spiritualisme et de la falsification du passé, à travers une mythologie de l'origine venant de l'intérieur, promue surtout par les leaders africains ${ }^{73}$. Dans son dernier recueil, Kezilahabi écrit explicitement sur la politique en employant un registre bas et transgressif pour insulter les puissants ou faire émerger des situations paradoxales avec ironie ou satire ${ }^{74}$, par exemple dans le poème qui donne le nom au recueil, Dhifa ${ }^{75}$, où une mouche s'approche du banquet pour manger la morve (kamasi, écrit ensuite kamaaasi, en transgressant aussi l'orthographe) du Bwana Mkubwa ("Grand Seigneur »), puis va sur la scène où les artistes jouent pour les participants du banquet et Akunja kiunoche/ Kwa shibe anya kabisa/ Ni dhifa ("Serre les hanches/ Pour déféquer à satiété/ C'est ça le banquet »).

Si nous considérons la sélection de poèmes que nous avons pu analyser, la poésie de Mudekereza semble dépourvue de ces éléments parodiques ou satiriques. Mais cela ne veut pas dire qu'elle soit également dépourvue d'aspects socio-politiques. Par exemple, le poème Pima tuyambe, bien qu'il ne traite pas de façon explicite de thématiques socio-politiques, pourrait laisser place à d'autres niveaux d'interprétation au-delà de sa dimension individuelle, qui révèlent l'attention de l'auteur à la vie sociale de sa ville, et à la crise économique et politique du Katanga. Le poème Pima uyambe (littéralement : "ose, et tu vas chier»; "ose, et tu en auras pour ton compte ») est, en fait, une expression populaire de Lubumbashi qui, à partir des années de la crise économique, a commencé à être écrite sur les maisons dont la vente était l'objet d'un conflit familial. La phrase en swahili, ou son équivalent en français " cette maison n'est pas à vendre ", est un moyen d'affirmer une opposition à la vente d'un patrimoine matériel et mémorial de la famille, et de dissuader les éventuels acheteurs. Ce phénomène est non seulement l'indice de la crise économique du Katanga liée au déclin de l'industrie minière, mais également de la désagrégation socio-familiale qui s'en est suivie. Il a frappé aussi l'imagination d'un artiste visuel de Lubumbashi, Georges Senga, lequel a créé un projet photographique intitulé «Cette maison n'est pas à vendre et à vendre ${ }^{76} »$.

Ni Kezilahabi ni Mudekereza ne souhaitent rendre la langue ou le style plus élégants, ou le message plus simple; leurs créations témoignent d'une forte unité poétique, qui renvoie constamment l'une à l'autre. Pour eux, la poésie n'est pas une manière plus haute de dire les choses, comme le soulignait Heidegger ${ }^{77}$. La poésie est un dire à l'écoute ${ }^{78}$, elle fait partie de notre expérience; en ce sens, Kezilahabi parle de event lived (" événement vécu ») qui peut devenir living event (« événement vivant») ${ }^{79}$. La poésie pour Heidegger est un dire originel ${ }^{80}$ qui dit en écoutant, et, donc asservit et libère ${ }^{81}$. La poésie, en fait, n'a pas besoin de l'extase pour créer un écart cognitif chez le lecteur. La poésie de Kezilahabi et de Mudekereza est comme une rupture, une fracture 
non seulement linguistique ou esthétique, mais aussi sensible, produisant cet écart cognitif qui fait émerger dans notre corps une nouvelle conscience, une vérité que la poésie nous pousse à accueillir. La vérité ne peut que coïncider avec la libération, comme l'affirment Heidegger et Kezilahabi ${ }^{82}$. C'est à cette libération qu'aspire Mudekereza. Quand Kezilahabi reste monolingue et pousse le langage poétique swahili vers le registre quotidien utilisé à l'oral, Mudekereza va dans la même direction, mais en déployant la Babel linguistique de Lubumbashi.

\section{Naviguer parmi les langues}

Mudekereza aime "naviguer parmi les langues ${ }^{83}$ ». Il écrit en français, en swahili et parfois en lingala - il convient de préciser que son swahili subit l'influence de la variété de Bukavu, au Kivu, d'où vient sa famille ${ }^{84}$.

Le poème Langues est particulièrement intéressant à cet égard. Il présente plusieurs langues : du swahili et du français en strophes distinctes, et des vers en lingala dans une strophe en swahili.

\begin{tabular}{|l|l|}
\hline Zunguluka tukutane & Fais un tour, pour que nous nous rencontrions \\
\hline Ngambo na ngambo aina mambo & D'un côté et de l'autre il n'y a pas de problème \\
\hline Kwetu ni kwenu & Chez nous c'est chez vous ${ }^{85}$ \\
\hline & Il n'y a plus de murs à escalader \\
\hline & Plus de barbelé, plus de gendarmes \\
\hline & Juste une main qui se tend \\
\hline Aema sasa, sema yote & À la mine de mon stylo \\
\hline Hadisi ya nkambo, habari za inchi & Les contes des grands-parents, les nouvelles du pays \\
\hline Maisha, matumaini & Lexique, vocabulaire, étymologie \\
\hline Boka na mateso & Parle maintenant, dis tout \\
\hline La vie, les espoirs \\
\hline & La peur et les tourments \\
\hline & Le silence qui rebondit dans nos regards \\
\hline & Pris au piège, étouffé dans l'air \\
\hline & Lâche un dernier cri \\
\hline & Je capte, caresse, tords et écris \\
\hline & Stylistique défigurée \\
\hline & Je vais pleurer avec tes larmes \\
\hline
\end{tabular}




\begin{tabular}{|l|l|}
\hline Nitacheka na mate yako & Je vais rire avec ta salive \\
\hline Tukatiyane tonge ya bugali & Qu'on se partage la bouchée de bugali86 \\
\hline Karibu, karibu kwangu & Bienvenue, bienvenue chez moi \\
\hline Maloba ya sukali & Les mots sucrés \\
\hline Bolingo ya malasi & L'amour parfumé \\
\hline Tokolangwa?87 & Allons-nous nous enivrer ? \\
\hline & Et comme dans un embouteillage \\
\hline & Les mots s'arrêtent \\
\hline & Toutes les langues sont au feu rouge \\
\hline & Les quitter et marcher à pied \\
\hline & Main dans la main \\
\hline
\end{tabular}

Le poète fait ici appel à l'hospitalité des langues, qui deviennent un lieu à habiter, «Bienvenue, bienvenue chez moi », et sont capables de confluer l'une dans l'autre, Kwetu ni kwenu. «Chez nous c'est chez vous » n'est pas une simple hospitalité, mais le fait de confluer d'un lieu habité vers l'autre, quasiment comme un mot qui entre dans une langue et se comporte comme chez soi. Seule l' "étymologie» fait remonter la trace. De cette manière, les deux langues persistent dans l'écriture de Mudekereza et, malgré la conscience de la séparation et de la disparité, l'une accueille la signification de l'autre, comme nous l'avons vu ci-dessus dans le texte Funga macho, liya. Dans le poème Langues, ce n'est pas simplement un lieu ou une langue qui conflue dans l'autre, mais les mêmes corps se fondent : " Je vais pleurer avec tes larmes /je vais rire avec ta salive ». Le poète met ici en acte une suspension de l'autre à travers l'action déflagrante de la poésie - "Juste une main qui se tend/ À la mine de mon stylo [...] Stylistique défigurée » - et à l'hospitalité du corps et de la langue. Si l'usage de deux langues n'est pas à prendre comme une manifestation sociopolitique, la suspension de l'autre n'est pas un acte violent de suppression identitaire, mais un geste d'accueil et d'acceptation totale, une dualité que l'art et l'amour (éros) arrivent à tenir ensemble sans prévarication. Dans ce texte, donc, l'érotisme et la poésie sont les éléments déflagrants qui permettent à deux mondes d'être ensemble. Dans le poème Langues, éros et poésie coïncident. C'est pour cette raison que les langues restent séparées dans les strophes, pas confondues, pour signaler deux identités distinctes et, même, l'échec de la promesse (en amour) et de la potentialité de fusion (dans l'art). Selon le philosophe Georges Bataille ${ }^{88}$ l'amour et l'éros promettent une fusion temporaire et, donc, illusoire. Dans la passion prend corps l'image de la fusion, mais c'est une promesse en partie vaine.

Cet effort va au-delà des langues et fait appel directement à la force érotique et accueillante du corps, de la chair, et à celle générative de la poésie. Autrement dit, le poète s'en remet à la transgression linguistique propre à la poésie, une transgression du 
langage ordinaire, et l'exalte à travers l'usage de plusieurs langues et d'un registre corporel. C'est en ce sens que Kezilahabi parle de poésie au-delà de la langue, une poésie capable de traverser les barrières spatio-temporelles ${ }^{89}$ et d'atteindre ainsi l'« audelà ", ce beyond qui est caractéristique de l'art. Cela permet, au moins dans son moment plus extatique, une suspension du principe de non contradiction, une suspension du " deux », de la différence, qui est nécessaire pour surmonter la douleur de la solitude, comme dit Bataille, pour tenter une fusion et essayer de traverser audelà de l'existence individuelle. C'est pour cela que la stylistique est mise à mal, afin que la poésie échappe au principe d'individuation, au rapide jugement conclusif, se manifestant au contraire comme éternellement présente.

La liberté est individuelle, comme Patrick Mudekereza le mentionne dans notre entretien (23/9/2018), parce que ni lui ni sa poésie ne représentent l'expression du Congo ou de Lubumbashi. Il ne se sent investi d'aucun mandat. Cela pourrait paraître comme l'aveu d'une limitation, mais pas du tout. Cela nous indique autre chose : avec cette déclaration de liberté et son lyrisme charnel, Mudekereza nous ramène au corps, non seulement comme référent, mais au corps de la poésie, soit avec son usage du multilinguisme, soit (et ici la transgression s'érige en protection de la poésie même) comme un corps nu à ne pas interpréter et violer.

La poésie se présente dans sa nudité et dans son action, pas comme symbole, mais comme charnalité. Mettant en garde contre les risques d'une interprétation des œuvres détachée du caractère expérimental de l'art, Susan Sontag écrivait, déjà dans les années 1960, dans son essai On style :

A work of art encountered as work of art is an experience, not a statement or an answer to a question. Art is not only about something; it is something. A work of art is a thing in the world, not just a text or mementary on the world ${ }^{90}$.

Et concluait dans Against Interpretation :

What is important now is to recover our senses. We must learn to see more, to hear more, to feel more. [...] In place of a hermeneutics we need an erotics of art $^{91}$.

C'est précisément ce que Mudekereza nous communique avec sa poésie et ses textes créatifs : nous avons besoin d'un autre scénario qui rende plus digestes et confortables l'art et la réalité. Mudekereza nous invite à faire l'expérience de l'art à la première personne, c'est pour cela qu'il nous bouscule avec l'emploi de plusieurs langues, exprimant ainsi la réalité urbaine de Lubumbashi, et c'est pour cela que les éléments de la corporalité ne sont pas seulement « seins », «bras », « dents » et " yeux », mais aussi "sueur", "urine», "chier». En d'autres mots, en faisant appel à la force de la transgression, le poète dans une manière a-idéologique construit sa poétique sur la base de son expérience corporelle, qui est aussi l'expérience du lecteur. Comme l'écrit Kezilahabi dans un essai sur la poésie swahilie classique et moderne: "Un poète compétent essaiera d'utiliser tous ses moyens pour que le lecteur voie l'image de ce qui a été dit [dans sa poésie] ; il peut exciter le corps ou faire en sorte que le lecteur sente l'odeur du sang (par exemple) ${ }^{92}$. " Le poète, partant de l'événement vécu, event lived dirait Kezilahabi ${ }^{93}$, peut atteindre l'« au-delà ", le beyond ${ }^{94}$ et se faire expérience de tous. C'est cet «au-delà » si charnel auquel Rimbaud aspirait, au mois de mars 1870, dans le poème « Sensation» :

Par les soirs bleus d'été, j'irai dans les sentiers,

Picoté par les blés, fouler l'herbe menue :

Rêveur, j'en sentirai la fraîcheur à mes pieds.

Je laisserai le vent baigner ma tête nue.

Je ne parlerai pas, je ne penserai rien : 
Mais l'amour infini me montera dans l'âme,

Et j'irai loin, bien loin, comme un bohémien,

Par la Nature, - heureux comme avec une femme ${ }^{95}$.

Bien sûr, cette potentialité d'émerger dans le corps du lecteur et cette dimension d'universalité propre à toute la poésie resteront castrées tant que les textes de Mudekereza resteront dans un tiroir ou publiés seulement dans des articles académiques, ne trouvant pas de lecteurs, mais tout au plus des critiques littéraires même la plupart des poèmes cités ont initialement été lus dans un cercle, celui de Libr'Écrire, collectif d'écrivains qui a existé à Lubumbashi entre 2008 et 2010. Comme le dit George Steiner, c'est le lecteur qui incarne le texte, le critique le regarde avec distance $^{96}$.

\section{Conclusion}

Ces textes poétiques de Patrick Mudekereza, bien qu'en nombre réduit, ont leur importance dans la réflexion poétique et dans l'esthétique de la poésie swahilie, en offrant, comme nous l'avons démontré, une production lyrique qui se charge de transgresser et mettre à nu la chair et le désir. Dans cette perspective, sa production s'inscrit dans la continuité avec l'œuvre d'un autre outsider de la littérature swahilie, Euphrase Kezilahabi, qui a épaté les conservateurs depuis la fin des années 1960 avec ses incessantes réformes poétiques, un héritage qui est resté isolé dans son pays, la Tanzanie, où il n'a été prolongé par aucun poète ${ }^{97}$. Les deux auteurs donnent voix à travers leur écriture, transgressive du point de vue de la langue, au plaisir sensuel, qui est aussi cognition. À cet égard il nous semble productif d'interpréter la poésie de Mudekereza à la lumière de la tradition littéraire swahilie, parce que cela pourrait donner un nouvel élan à la recherche sur le vers libre swahili, sur le style et sur l'esthétique en général. C'est une production qui, à notre avis, mérite d'être diffusée largement et traduite en d'autres langues.

\section{BIBLIOGRAPHIE}

Flavia AIELlo \& Roberto GAUDIOSo, « Sando Marteau : il cantore di Lubumbashi », Kervan, n²3, 2019, p. 7-28.

Jacques BAKASANDA, « La poésie au Katanga (1989-2009) », Études littéraires africaines, n² 27, 2009, p. 36-45.

Georges BATAILLE, L'erotismo, Milano, ES, 1997.

Donatien DIBWE DIA MWEMBU, «Le passé colonial et postcolonial dans les débats du projet

"Mémoires de Lubumbashi" ", dans Afrique et Occident : Mémoires et identités dans la Région des

Grands Lacs, C. Carbone et R. Giordano (dir.), Paris, L'Harmattan, 2009, p. 117-132.

Donatien DiBWE DIA MWEMBU \& Claude MWILAMBWE MWENDE, Musique urbaine au Katanga. De Malaika à

Santu Kimbangu, B. Jewsiewicki (éd.), Paris, L'Harmattan, 2003, p. 87-112. 
Étienne DAMOME \& Emmanuel KAMBAJA, Le Kiswahili dans les médias audiovisuels de Lubumbashi : usages et représentations, Kinshasa, Éditions CEDI, 2012.

Johannes FABIAN, Language and Colonial Power: the Appropriation of Swahili in the Former Belgian Congo 1880-1938, Cambridge, Cambridge University Press, 1986.

Aurélia FERRARI, Marcel KALUNGA \& Georges MULUMBWA, Le Swahili de Lubumbashi. Grammaire, textes, lexique, Paris, Karthala, 2014.

Roberto GAUDIOSO, « Transferring and Rewriting Freedom in Euphrase Kezilahabi », NJAS 24(1), 2015, p. 63-89.

Roberto GAUDIOSO, « A Literary Approach to Avoiding Objectification of the Text: Reading Kezilahabi and Beyond ", AIOO, n 77, 2017, p. 3-32.

Roberto GAUDIOSo, The voice of the text and its body. The continuous reform of Kezilahabi's poetics. Cologne, Rüdiger Köppe Verlag, 2019.

Martin HEIDEGGER, In cammino verso il linguaggio, Milan, Mursia, 1973.

Jean de Dieu KARANGWA, Le Kiswahili dans l'Afrique des Grands Lacs : contribution sociolinguistique, thèse de doctorat, INALCO, Paris, 1995.

Euphrase KEZILAHABI, « Ushairi wa Mapokeo na Wakati Ujao », dans Uandishi wa Tanzania, Nairobi/ Kampala/Dar-es-Salaam, East African Literature Bureau, 1976, p. 1-11.

Euphrase KEZILAHABI, African Philosophy and the Problem of Literary Interpretation, PhD thesis, Madison, University of Wisconsin, unpublished, 1985.

Euphrase KEZILAHABI, Dhifa, Nairobi, Vide Muwa, 2008.

Maëline LE LAY, «Les langues d'écriture à Lubumbashi : une littérature diglossique ? », Études littéraires africaines, $\mathrm{n}^{\circ} 27,2009$, p. 55-64.

Maëline LE LAY, La parole construit le pays. Théâtre, langues et didactisme au Katanga (République Démocratique du Congo), Paris, Honoré Champion, 2014.

Maëline LE LAY, « De la rumeur de la ville à la voix de l'Autorité : les écrits en swahili à Lubumbashi », Journal des Africanistes, nº 83(1), 2014, p. 14-37.

Maëline LE LAY, « Joseph Kiwele in Elisabethville (1946-1961) and the Birth of an Urban Culture in the Colonial Era », dans A Blueprint for Toads and Snakes, S. Baloji \& V. Van Velsen (éd.), Amsterdam, Framer Framed, 2018, p. 16-26.

Kapambwe LUMBWE, « Indigenous mfunkutu and contemporary ubwinga (wedding) music of the Bemba-speaking people of Zambia: continuity and change ", Journal of the Musical Arts in Africa, $\mathrm{n}^{\circ}$ 10(1), 2013, p. 71-101.

Crispin MAALU-BUNGI, Les Inconnus de l'histoire. Introduction à la littérature créative en langues congolaises, Kinshasa, Éditions Mabiki, sous presse.

Crispin MAALU-BUNGI, « Written literature in Congolese languages: origin and principal genres », dans Beyond the Language Issue. The production, Mediation and Reception of Creative Writing in African Languages, A. Oed et U. Reuster-Jahn (éd.), Köln, Rüdiger Köppe Verlag, 2008, p. 33-40.

Antoine MUIKILU-NDAYE, Topique des représentations théâtrales en R. D. Congo de 1905 à 1960, Paris, Presses Académiques Francophones, 2014.

Mugyabuso M. MULOKOZI \& Tigiti S. Y. SENGo, History of Kiswahili Poetry [AD 1000 - 2000], Dar-esSalaam, Institute of Kiswahili Research, 1995. 
Nico NASSENSTEIN \& Paulin Baraka BOSE, Kivu Swahili texts and grammar notes, Muenchen, LINCOM, 2016.

Pius NGANDU-NKASHAMA, Littératures et écritures en langues africaines, Paris, L'Harmattan, 1992.

Pierre PETiT \& Georges MUlumbWa MUTAMBWA, « "La crise" : Lexicon and ethos of the second economy in Lubumbashi ", Afrika 75/4, 2005, p. 467-486.

Joël NORET \& Pierre PETIT, Mort et Dynamiques sociales au Katanga, Paris, L'Harmattan, 2011.

Arthur RIMBAUD, Tutte le poesie, Milan, Newton, 2007.

Alena RETTOVÁ, Chanter l'existence : la poésie de Sando Marteau et ses horizons philosophiques,

Středokluky, Zdeněk Susa, 2013.

Silvia RIVA, Nouvelle histoire de la littérature du Congo-Kinshasa, Paris, L'Harmattan, 2006.

Susan SONTAG, Against Interpretation and Other Essays, Londres, Penguin, 2009 [première edition en 1969].

George STEINER, “Critic”/“Reader”, New Literary History, vol. 10, n 3, 1979, p. 423-452.

Wilfred WHITELEY, Swahili. The Rise of a National Language, Londres, Methuen, 1969.

\section{NOTES}

1. Le swahili est parlé par environ neuf millions de locuteurs, principalement dans les territoires orientaux et sud-orientaux du pays. Jacques LECLERC, "Congo-Kinshasa », dans L'Aménagement linguistique dans le monde, Québec, TLFK, université Laval, 24 juin 2009. http:// www.axl.cefan.ulaval.ca/afrique/czaire.htm. Le swahili dispute le statut de première langue véhiculaire au lingala, compris par la majorité des Congolais, y compris ceux de l'Est swahiliphone.

2. Sous la colonisation belge, l'éducation était confiée aux missionnaires qui éduquaient et évangélisaient les élèves dans leurs langues, ce qui a donc pu conduire l'amorce d'une production créative en langues locales, pour la plupart exercices d'écriture scolaire destinés à rester dans l'enceinte de la classe. Il faut aussi préciser que, quoiqu'il y eût dans les années 1920-1930 quelques concours littéraires ouverts dans différentes langues congolaises, le champ littéraire s'est structuré en français, dès la période coloniale, et ce pour différentes raisons. Il s'agissait pour les Belges, de se doter d'une vitrine coloniale littéraire et artistique, pour convaincre la population métropolitaine de l'intérêt de cette colonie, et pour rivaliser avec les puissances impériales voisines, notamment la France. Voir Maëline LE LAY, La parole construit le pays. Théâtre, langues et didactisme au Katanga (République Démocratique du Congo, Paris, Honoré Champion, 2014. Dans le théâtre missionnaire au Congo, par exemple, il y avait une prédominance du français dans les pièces jouées mais aussi écrites dans les missions. Voir Antoine MUIKILU-NDAYE, Topique des représentations théâtrales en R. D. Congo de 1905 à 1960, Paris, Presses Académiques Francophones, 2014.

3. Crispin MAALU-BUNGI, « Written literature in Congolese languages: origin and principal genres ", dans Beyond the Language Issue. The production, Mediation and Reception of Creative Writing in African Languages, edited by Anya Oed and Uta Reuster-Jahn, Köln, Rüdiger Köppe Verlag, 2008, p. 33.

4. Ibid., p. 34.

5. À partir de l'Indépendance, en continuité substantielle avec la période coloniale, la langue officielle au Congo-Kinshasa est le français, médium de l'administration et de l'éducation, suivi des langues nationales, à savoir le ciluba, le kikongo, le lingala et le swahili. Les nombreuses 
autres langues africaines n'avaient pas et n'ont pas toujours de statut. Voir Jean de Dieu KARANGWA, Le Kiswahili dans l'Afrique des Grands Lacs: contribution sociolinguistique, thèse de doctorat, INALCO, Paris, 1995, p. 109.

6. Silvia RIVA, Nouvelle Histoire de la littérature du Congo-Kinshasa, Paris, L'Harmattan, 2006, p. 336.

7. Sous "l'Authenticité » de Mobutu ont paru quelques plaquettes de littérature en langues congolaises (parues aux éditions Mont-Noir, pour la plupart), puis ce n'est qu'après plusieurs décennies que l'on a pu voir l'essor timide et marginal de quelques parutions en langues nationales, par exemple récemment grâce aux éditions Nzoi (http://www.editions-nzoi.org/) et aux éditions Mabiki (http://www.mabiki.net/), fondées par Bienvenu Sene Mongaba.

8. Voir Pius NGANDU-NKASHAMA, Littératures et écritures en langues africaines, Paris, L'Harmattan, 1992, p. 381-382, et Crispin MAALU-BUNGI, Les Inconnus de l'Histoire. Introduction à la littérature créative en langues congolaises, Éditions Mabiki, Kinshasa, sous presse.

9. La première pièce théâtrale où le swahili a été employé est Chura na Nyoka (Le Crapaud et le Serpent, 1952), publiée à Elizabethville (nom de l'actuelle ville de Lubumbashi), une comédie musicale inspirée d'un conte kongo. Elle fut écrite en swahili, français et kibemba par Joseph Kiwele, organiste, compositeur, enseignant de musique et homme politique (élu député provincial, puis nommé ministre de l'Éducation dans le gouvernement provincial du Katanga, il composera aussi l'hymne national du Katanga en 1960 (Voir Crispin MAALU-BUNGI, Les Inconnus de l'Histoire, op.cit., p. 2 ; Maëline LE LAY, « Joseph Kiwele in Elisabethville (1946-1961) and the Birth of an Urban Culture in the Colonial Era ", dans A Blueprint for Toads and Snakes, S. Baloji \& V. Van Velsen (éd.), Amsterdam, Framer Framed, 2018, p. 16-26.

10. La troupe théâtrale Mufwankolo, par exemple, l'une des plus anciennes et fameuses troupes de théâtre populaire au Congo, improvise toutes ses pièces à partir d'un bref canevas en français. Seules l'introduction (utangulizi) et la morale de l'histoire (mafundisho) sont écrites par l'auteur dans une forme qui se rapproche du swahili standard. Voir Maëline LE LAY, La parole construit le pays, op. cit., p. 24-27.

11. Rehema. Mnara ya Baba na Mama (Rehema. Soutien de père et mère, 1973) de N'sanda Wamenka, publié à Lubumbashi (voir MAALU-BUNGI, Les Inconnus de l'Histoire, op. cit., p. 3).

12. Jean-Robert Kasele Laisi Watutwa (1946-2004), né à Kamituga (Sud-Kivu) en 1946, a été professeur et premier recteur de l'Université Pédagogique Nationale de Kinshasa (RDC). Il a aussi vécu longuement en Belgique où il a lancé sa maison d'édition (les éditions du Pangolin). Il est également l'auteur de plusieurs œuvres littéraires (poésie, prose et théâtre) en français. Sa production poétique en swahili est constituée de Bibi (Épouse, 1986) et Simamemi Wakongomani (Levez-vous, Congolais, 1999). Le premier recueil est composé de quinze textes à thématique amoureuse en swahili, français et kirega, sa langue maternelle. La deuxième plaquette comprend onze poèmes en swahili, très patriotiques, dédiés aux victimes des massacres de Kasika et Makobola (voir MAALU-BUNGI, Les Inconnus de l'Histoire, op. cit., p. 5).

13. Charles Djungu-Simba, né en 1953 à Kamituga, est professeur à la Faculté de Lettres de l'Université Pédagogique Nationale de Kinshasa. Il compte aujourd'hui parmi les auteurs francophones congolais les plus prolifiques (nouvelles, romans, poésie, biographies, théâtre). En tant qu'écrivain swahiliphone, il a publié deux recueils poétiques, Kongo Yetu (Notre Congo, 2000) et BORA UZIMA na Mashairi mengine (Vivre pleinement et d'autres poèmes, 2019). Les vers de DjunguSimba comprennent aussi des thèmes nationaux, comme les invocations à l'unité du pays et à ses héros (Kimpa Vita, Lumumba, Mulele etc.), la mémoire des atrocités commises par les esclavagistes, les Belges, et plus récemment les Ruandais, des critiques envers Mobutu et les Kabila, etc. Voir MAALU-BUNGI, Les Inconnus de l'Histoire, op. cit., p. 11; http://mukanda.univlorraine.fr/.

14. Mugyabuso M. MULOKOZI \& Tigiti S. Y. SENGO, History of Kiswahili Poetry [AD 1000 - 2000], Dar-esSalaam, Institute of Kiswahili Research, 1995, p. 22. 
15. Aurélia FERRARI, Marcel KALUNGA \& Georges MULUMBWA, Le Swahili de Lubumbashi. Grammaire, textes, lexique, Paris, Karthala, 2014, p. 5.

16. La standardisation du swahili fut entreprise par l'East African Swahili Committee. Cet organisme commença à se réunir à partir de 1930, mais sa constitution avait déjà été évoquée au cours d'une conférence organisée à Dar-es-Salaam par le gouverneur du Tanganyika en 1925. L'objectif de la conférence était la sélection d'une langue pouvant servir de langue véhiculaire pour tout le territoire colonial. Pour cela fut choisi le swahili, pour lequel se révéla nécessaire une standardisation par une orthographe commune et une seule forme dialectale de référence, le kiunguja de Zanzibar. Voir Wilfred WHITELEY, Swahili. The Rise of a National Language, Londres, Methuen, 1969, p. 79-80.

17. À cette époque, la langue swahilie est employée non seulement comme moyen d'évangélisation et d'instruction par les missionnaires catholiques dans l'est du Congo, mais aussi comme langue véhiculaire dans le cadre d'une «belgianisation » d'Élisabethville (fondée vers 1910 ; aujourd'hui Lubumbashi) et du personnel de l'Union minière du Haut-Katanga, favorisée par l'administration et les forces économiques pour remplacer le kitchen-kafir et réduire la dépendance face aux compagnies d'Afrique du Sud. La généralisation du swahili était d'autant plus nécessaire que l'on passa de l'exploitation de faible technologie des minéraux (en particulier le cuivre, shaba en swahili) à une production qui requérait une population ouvrière plus stable et plus qualifiée, recrutée de plus en plus dans le Nord-Est (Stanleyville, actuelle Kisangani ; Kivu ; Katanga du Nord), où le swahili était l'instrument de communication. Voir Johannes FABIAN, Language and Colonial Power: the Appropriation of Swahili in the Former Belgian Congo 1880-1938, Cambridge, Cambridge University Press, 1986, p. 92-106.

18. Certains habitants de Lubumbashi ont une compétence passive de la langue de référence identitaire, ou ne la connaissent pas. Voir FERRARI, KALUNGA \& MULUMBWA, op. cit., p. 8.

19. FERRARI, KALUNGA \& MULUMBWA, op. cit., p. 6.

20. À Lubumbashi le swahili est employé dans divers genres chantés, et il est lié en particulier au développement de la chanson moderne katangaise, avec des auteurs très fameux comme Edouard Masengo Katiti, Losla Abelo et Mwenda wa Bayeke. En outre, il est le moyen d'expression du théâtre populaire lushois (les deux troupes plus connues sont la susmentionnée Troupe Mufwankolo et le Collège Nzembela). Pour en savoir plus sur le théatre populaire katangais, voir Maëline LE LAY, La parole construit le pays, op. cit.

21. Voir MAALU-BUNGI, Les Inconnus de l'Histoire, op. cit., p. 1.

22. Voir FERRARI, KALUNGA \& MULUMBWA, op. cit., p. 130.

23. Lubumbashi, interview du 23/9/2018.

24. Nous remercions l'anthropologue Edoardo Quaretta pour nous avoir introduits au programme ACCELERE! (https://devtracker.dfid.gov.uk/projects/GB-1-203154). Pendant son travail de terrain à Lubumbashi, le chercheur a suivi des leçons de swahili dans des écoles de la périphérie, et a parlé avec des enseignants ; c'était en 2017, année où beaucoup d'entre eux ont pris part à des cours de didactique de swahili bora. Il a noté de nombreuses plaintes dues au fait de devoir enseigner une langue très distante de celle parlée au quotidien à Lubumbashi.

25. La presse en swahili, jadis présente à l'époque coloniale, est aujourd'hui inexistante. La langue swahilie, au contraire, est présente aussi bien dans le secteur radiophonique (national et régional), que dans celui des télévisions locales (la chaîne nationale transmet principalement en français). Au Katanga, il n'y a pas de radios ou télévisions qui diffusent uniquement en swahili ; toutefois, le swahili est très présent dans les médias, spécialement à partir de la libéralisation du marché audiovisuel dans les années 1990. Depuis ce moment, le secteur privé de la radiotélévision est en forte croissance (voir Etienne DAMOME \& Emmanuel KAMBAJA, Le kiswahili dans les médias audiovisuels de Lubumbashi : usages et représentations, Kinshasa, Editions CEDI, 2012, p. 20-26. À Lubumbashi, certaines chaînes comme Nyota, Mwangaza et Jua diffusent les émissions 
culturelles en swahili local (dit «swahili facile»). Le journal télévisé est diffusé en français, swahili standard et swahili de Lubumbashi (voir FERRARI, KALUNGA \& MULUMBWA, art. cit., p. 132).

26. Voir Maëline LE LAY, « De la rumeur de la ville à la voix de l'Autorité : les écrits en swahili à Lubumbashi ", Journal des Africanistes, $\mathrm{n}^{\circ}$ 83, 2014, p. 14-37.

27. La recherche s'est déroulée aux mois de septembre et octobre 2018. Le projet, financé pour la période 2017-2020 par le ministère de l'Éducation, de l'Université et de la Recherche italien, a été coordonné par l'équipe de l'Université de Calabre (dirigée par R. Giordano), avec la participation de deux unités italiennes, à l'Université de Milan (unité dirigée par S. Riva) et à l'Université de Naples «L'Orientale » (unité dirigée par F. Aiello), et d'une unité à l'Université de Lubumbashi (dirigée par D. Dibwe Dia Mwembu).

28. Sando Marteau est un artiste très connu à Lubumbashi en tant que comédien dans la Troupe Mufwankolo, conteur et chanteur-compositeur. Ses compositions s'inscrivent dans la lignée de la chanson populaire swahilie de Lubumbashi (Mwenda wa Bayeke, E. Msengo Katiti etc.), tout en développant un style musical et poétique très personnel.

29. Voir Alena RETTOVÁ, Chanter l'existence : la poésie de Sando Marteau et ses horizons philosophiques, Středokluky, Zdeněk Susa, 2013.

30. Interview de S. Marteau, 28/9/2018, Lubumbashi.

31. Sando Marteau n'emploie jamais la langue française dans ses textes, ni le français littéraire, ni les expressions du quotidien typiquement utilisées sous forme de code-switching et code-mixing dans le théâtre populaire de Lubumbashi. L'auteur crée un nouveau langage poétique swahili en exploitant les potentialités expressives dérivant de la dimension fluide, non standardisée du swahili à Lubumbashi, précisément ce que ses locuteurs considèrent comme un signe d'infériorité linguistique. Aussi l'orthographie employée par Sando Marteau est-elle révélatrice du statut essentiellement oral du swahili local et de l'absence d'un système d'écriture standardisé (FERRARI, KALUNGA \& MULUMBWA, art. cit., p. 13).

32. Flavia AIELlo \& Roberto GAUdioso, «Sando Marteau : il cantore di Lubumbashi », Kervan, $\mathrm{n}^{\circ} 23$, 2019, p. 7-28.

33. Ibid., p. 23-24.

34. Un des cas rares où on note l'influence de l'orthographie française : le diphtongue « oi » se lit « ua ». Wa foisi correspond, en fait, à wafuasi (suiveurs) en swahili standard.

35. MAALU-BUNGI, Les Inconnus de l'Histoire, op. cit., p. 6.

36. Jano BAKASANDA, «La poésie au Katanga (1989-2009) », Études littéraires africaines, $\mathrm{n}^{\circ} 27,2009$, p. 36-45.

37. Voir MAALU-BUNGI, Les Inconnus de l'Histoire, op. cit., p. 7.

38. NGANDU-NKASHAMA, op. cit., p. 381.

39. BAKASANDA, « La poésie au Katanga (1989-2009) », art. cit.

40. Ibid., p. 39.

41. Ibid., p. 41.

42. BAKASANDA, art. cit., p. 45.

43. LE LAY, op. cit., 2009, p. 57.

44. Nom d'un lieu en RDC, mais qui peut aussi indiquer le pays du peuple sanga (Basanga).

45. Le mot «Kisanga » peut avoir plusieurs significations : il est le nom d'un lieu en Tanzanie, parfois on lui donne le sens de « île », et il renvoie aussi à la langue sanga des Basanga.

46. Les paysans ont l'habitude de quitter leur village et d'aller passer plusieurs jours aux champs pour avoir le temps de travailler plus longtemps. Après, ils retournent au village.

47. Notre étude se fonde sur un nombre limité de textes reçus de Patrick Mudekereza, dont certains datent de plus de dix ans, il s'agit donc de résultats partiels.

48. Le verbe waza en swahili signifie "pense", "imagine». Pour des informations sur les activités du centre, voir https://www.centredartwaza.org/. 
49. Patrick Mudekereza a par exemple été invité avec l'artiste visuel Sammy Baloji à une résidence artistique au Musée Royal de l'Afrique Centrale et à l'Université de Gand, en Belgique. $\mathrm{Au}$ cours de la résidence, ils ont produit diverses créations artistiques visuelles/verbales, en dialogue avec une équipe scientifique multidisciplinaire. Et ils ont choisi de baptiser le projet «Congo Far West », stimulant ainsi une réflexion sur le rapport entre les collections du musée et la République Démocratique du Congo d'aujourd'hui. De ce projet sont nées une publication intitulée Congo Far West (Silvana Editoriale, Milano 2011) et une exposition présentée au MRAC (11 mai - 4 septembre 2011).

50. Choquer ou ku-choquer (du français choc) est un nouveau verbe utilisé au début des années 1990 à Lubumbashi pour décrire l'action d'une personne qui chaque matin sort déterminée à trouver une activité de travail qui puisse soutenir sa famille (voir Pierre PETIT \& Georges MULUMBWA MUTAMBWA, "La crise": Lexicon and ethos of the second economy in Lubumbashi ", Afrika 75/4, 2005, p. 467-486; 474). Le terme semble être né parmi les Congolais excavateurs clandestins de diamants au Congo et Angola, et il est employé aussi au Kasaï oriental pour les excavateurs journaliers de diamants, se référant à la force physique et à la rapidité nécessaires pour ce travail. Il fait partie d'un nouveau répertoire d'expressions en français et swahili qui soulignent le courage et l'énergie mis à la recherche quotidienne de travail et de subsistance dans le contexte de l'économie informelle générée par la crise socio-économique, qui a démantelé le système de travail salarié (kazi ou kaji) autour duquel s'était développée la ville de Lubumbashi, en devenant un élément fondamental de l'identité collective urbaine. Voir Donatien DIBWE DIA MWEMBU, « Le passé colonial et postcolonial dans les débats du projet "Mémoires de Lubumbashi", dans Afrique et Occident: Mémoires et identités dans la Région des Grands Lacs, C. Carbone et R. Giordano (dir.), Paris, L'Harmattan, 2009, p. 117-132 ; 123.

51. Interview du 23/9/2018.

52. Pour donner un aperçu de la poétique de Jean Genet (Paris 1910-1986), citons par exemple son court-métrage Un Chant d'Amour (accessible en open source) ou ses romans Notre-Dame-des-Fleurs (1944), Querelle de Brest (1947), Pompes funèbres (1948), etc.

53. Uhuru veut dire aussi indépendance.

54. Le verbe -panda signifie aussi "planter, ensemencer", l'expression peut être donc aussi traduite « ensemençons l'un l'autre».

55. Le terme fait allusion aussi au fluide séminal.

56. Majiba veut dire aussi « seins ».

57. Euphrase KEZILAHABI, Karibu ndani [Bienvenu], Dar es Salaam, Dar es Salaam, University Press, 1988.

58. À la différence de sa poésie dans les années 1960 et 1970, où, par exemple, le sang est très présent. Voir Roberto GAUDIOSO, The voice of the text and its body. The continuous reform of Kezilahabi's poetics, Cologne, Rüdiger Köppe Verlag, 2019.

59. Euphrase KEZILAHABI, Dhifa [Banquet], Nairobi, Vide Muwa, 2008.

60. Ibid., « La femme du Ministre (Chant nuptial)», p. 13.

61. Ibid., « Existence », p. 10.

62. Ibid., « Inondation », p. 4.

63. Arthur RimBAUD, Tutte le poesie, Milan, Newton, 2007, p. 169.

64. Mudekereza se situe dans la tradition textuelle francophone aussi bien que swahiliphone. Grâce sans doute à ses études scolaires et universitaires, et ses intérêts culturels, il a une grande familiarité avec la littérature et la tradition textuelle francophones. Du côté du swahili, suite au statut de la langue décrit ci-dessus, il a surtout une connaissance des formes artistiques orales chantées.

65. Interview à Lubumbashi, 23/9/2018. 
66. Joël NORET \& Pierre PETIT, Mort et Dynamiques sociales au Katanga, Paris, L'Harmattan, 2011, p. 78.

67. Les chants kalindula à Lubumbashi sont chantés en swahili davantage, mais aussi en kibemba, kiluba et ciluba. Le chants zangazanga sont aussi en plusieurs langues : lingala, swahili, ciluba etc. (communication par e-mail du professeur Donatien Dibwe dia Mwembu, UNILU, 25/08/2020). Voir NORET \& PETIT, Mort et Dynamiques sociales au Katanga, op. cit., p. 59-149.

68. Le kalindula est une musique qui vient des Bemba qui vivent au nord de la Zambie et au sud du Katanga (RDC). Ces groupes commencèrent à être populaires dans les années 1970 à Lubumbashi ; il y a une nette différence entre le kalindula urbain et celui des villages. À Lubumbashi, le kalindula a toujours été perçu comme un simple élément folklorique, alors qu'en Zambie le genre a atteint un certain succès national (Donatien DIBWE DIA MWEMBU \& Claude MWILAMBWE MWENDE, Musique urbaine au Katanga. De Malaika à Santu Kimbangu, B. Jewsiewicki (dir.), Paris, L'Harmattan, 2003, p. 87-112; 110). Kapambwe Lumbwe rapporte que dans les années 1980 en Zambie le kalindula et le zamrock sont devenus populaires en ville, en compétition avec la rumba congolaise (bolingo music) : voir Kapambwe LUMBWE, «Indigenous mfunkutu and contemporary ubwinga (wedding) music of the Bemba-speaking people of Zambia: continuity and change », Journal of the Musical Arts in Africa, $\mathrm{n}^{\circ} 10$ (1), 2013, p. 71-101; 85.

69. DIBWE DIA MWEMBU et MWILAMBWE MWENDE, op. cit., p. 88.

70. Joël NORET \& Pierre PETIT, Mort et Dynamiques sociales au Katanga, op. cit., p. 78-149.

71. DIBWE DIA MWEMBU et MWILAMBWE MWENDE, op. cit., p. 87-111.

72. Ibid., p. 110.

73. Euphrase KEZILAHABI, African Philosophy and the Problem of Literary Interpretation, PhD thesis, University of Wisconsin, Madison (unpublished), 1985, p.357-358; Roberto GAUDIOSO, « Transferring and Rewriting Freedom in Euphrase Kezilahabi », NJAS, n 24(1), 2015, p. 63-89.

74. Voir Euphrase KEZILAHABI, Dhifa, Nairobi, Vide Muwa, 2008, notamment les poèmes Dikteta («Le dictateur», p. 31), Cha watu («Ce qui appartient aux gens», p. 38), Hatima ya watu (« La fin des gens, p. 44), ou encore là où il encourage explicitement à la responsabilité et à la liberté, comme dans Wimbo wa Unyago («Chant de l'initiation», p. 17), Binti («Fille», p. 35), Kwa watu wenye rangi (« Pour les personnes de couleur », p. 54).

75. «Banquet », 2008, p. 27.

76. Voir https://www.georgesengart.com/cette-maison-n-est-pas-a-vendre-et-.

77. Martin HEIDEGGER, In cammino verso il linguaggio, Milano, Mursia, 1973, p. 42.

78. Ibid., p. 71-74.

79. Pour approfondir, voir Roberto GAUDIOSO, «A Literary Approach to Avoiding Objectification of the Text: Reading Kezilahabi and Beyond», AIOO, $\mathrm{n}^{\circ}$ 77, 2017, p. 3-32; The voice of the text and its body. The continuous reform of Kezilahabi's poetics, Cologne, Rüdiger Köppe Verlag, 2019.

80. HEIDEGGER, op. cit., p. 35, 117-123.

81. HEIDEGGER, op. cit., p. 124.

82. Euphrase KEZILAHABI, African Philosophy and the Problem of Literary Interpretation, op. cit., p. 67.

83. Interview du 23/9/2018.

84. En tenant compte de la langue que Mudekereza a parlée à la maison pendant son enfance, il semble aisé d'expliquer les traits de son swahili plus proches de celui de Tanzanie, plutôt que comme un usage du kiswahili bora, modèle linguistique dont il n'a pas eu une vraie expérience à l'école, et auquel il n'aspire pas. Par exemple, dans le poème Langues plus bas, on trouve le mot bugali, qui ne présente pas de phénomènes de dévoisement $(g>k)$ et de transformation de $l$ en $r$ au contact avec le « $\mathrm{i}$ », typiques du swahili de Lubumbashi, où la forme est bukari (FERRARI, KALUNGA \& Mulumbwa, op.cit., p. 20-21). Dans ses textes, il y a aussi souvent des mots qui gardent le « $\mathrm{h}$ » initial, un son qui fait partie du répertoire de consonnes du swahili du Kivu, par exemple hadisi (en swahili standard hadithi; en swahili de Lubumbashi adisi/arishi), habari (en swahili de 
Lubumbashi abari), parfois omis en suivant le parler de Lubumbashi (Nico NASSENSTEIN \& Paulin Baraka BOSE, Kivu Swahili texts and grammar notes, Muenchen, LINCOM, 2016, p. 2).

85. L'expression kwetu, laquelle grammaticalement est la forme locative du possessif de la première personne du pluriel, est polysémique, elle peut indiquer « chez nous/chez moi », « dans mon lieu d'origine ", " dans mon pays ».

86. En swahili standard, ugal est le nom d'une nourriture très commune au Katanga, une pâte à base de farine de maïs ou manioc.

87. Les derniers trois vers sont en lingala.

88. Georges BatAILLE, L'erotismo, Milano, ES, 1997, p. 20-21.

89. Voir l'interview menée par Gaudioso en 2015 à Gaborone, Botswana.

90. Susan SONTAG, Against Interpretation and Other Essays, Londres, Penguin, 2009 [première édition en 1969].

91. Ibid., p. 14.

92. «Mshairi mashuhuri atajaribu kutumia vyombo hivi vyote ili aweze kumfanya msomaji aone picha ya mambo yanayozungumziwa mwake; anaweza kusisimua mwili au hata kumfanya msomaji asikie harufu ya damu au beberu (kwa mfano). », Euphrase Kezilahabi, «Ushairi wa Mapokeo na Wakati Ujao », dans Uandishi wa Tanzania, Nairobi/Kampala/Dar-es-Salaam, East African Literature Bureau, 1976, p. 1-11.

93. Euphrase KEZILAHABI, African Philosophy and the Problem of Literary Interpretation, op. cit., p. 220.

94. Kezilahabi dans Roberto GAUDIOSO, The voice of the text and its body. The continuous reform of Kezilahabi's poetics, Cologne, Rüdiger Köppe Verlag, 2019, p. 61-100.

95. Arthur Rimbaud, Tutte le poesie, Milano, Newton, 2007, p. 46.

96. George Steiner, “Critic"/“Reader”, New Literary History, vol. 10, n 3, 1979, p. 423-452.

97. Voir l'interview de Kezilahabi menée par Gaudioso en 2015, à Gaborone, Botswana.

\section{RÉSUMÉS}

L'article rend compte des recherches menées sur le terrain dans le cadre d'un projet d'intérêt national (PRIN) intitulé «Mobilité - stabilisation. Représentations congolaises et dynamiques sociales, au Congo et dans l'espace global », financé pour la période 2017-2020 par le ministère de l'Éducation, de l'Université et de la Recherche italien. Flavia Aiello et Roberto Gaudioso présentent des textes inédits de Patrick Mudekereza qu'ils ont rencontré en 2018 à Lubumbashi. Mudekereza n'a jamais publié ses poèmes dans des recueils poétiques, considérant son écriture essentiellement comme une pratique associée à son travail dédié à l'art. Engagé dans plusieurs formes d'écriture, créative et discursive (cette dernière en français ou anglais), l'artiste luhois semble avoir un rapport très particulier et intime à la poésie, qu'il pratique de manière intermittente et dont il ne garde pas trace de façon systématique. Dans cet article on analysera six textes, dont trois (Funga macho liya, Pima tuyambe et Langues) composés durant les travaux du collectif d'écrivains Libr'Écrire né à Lubumbashi en 2007 ; deux ne sont pas datés (Buzuri tele et Mu pori ya binvuri [Dans le bois des ombres]), et un (Mankamanshi>Mboleo [Morve>Fertilisant]) est daté du 13 février 2018. Trois de ces compositions poétiques sont bi/multilingue, en français, swahili et lingala (Funga macho liya, Langues, Mankamanshi>Mboleo), les autres sont écrits en swahili. 
This paper reports on field research carried out as part of a Project of National Interest (PRIN) entitled "Mobility - Stabilisation. Congolese representations and social dynamics, in Congo and in the global space", funded for the period 2017-2020 by the Italian Ministry of Education, University and Research. Flavia Aiello and Roberto Gaudioso present unpublished texts by Patrick Mudekereza whom they met in Lubumbashi in 2018. Mudekereza never published his poems in poetry collections, considering his writing essentially as a practice associated with his work dedicated to art. Engaged in several forms of writing, both creative and discursive (the latter in French or English), the artist from Lubumbashi seems to have a very particular and intimate relationship with poetry, which he practises intermittently and of which he does not keep track systematically. In this article we will analyse six texts, three of which (Funga macho liya, Pima tuyambe and Langues) were composed during the work of the writers' collective Libr'Écrire born in Lubumbashi in 2007; two are undated (Buzuri tele and Mu pori ya binvuri [In the Wood of Shadows]), and one (Mankamanshi>Mboleo [Snot>Fertilising]) is dated 13 February 2018. Three of these poetic compositions are bi/multilingual, in French, Swahili and Lingala (Funga macho liya, Langues, Mankamanshi>Mboleo), the others are written in Swahili.

\section{INDEX}

Mots-clés : poésie en kiswahili, plurilinguisme, Lubumbashi, littérature congolaise, Libr'Écrire

Keywords : poetry in Kiswahili, plurilingualism, Lubumbashi, Libr'Écrire

\section{AUTEURS}

\section{FLAVIA AIELLO}

Université de Naples L'Orientale

ROBERTO GAUDIOSO

Université de Naples L'Orientale 